\title{
Automatic gray images colorization based on lab color space
}

\author{
Nidhal K. EL Abbadi ${ }^{1}$, Eman Saleem ${ }^{2}$ \\ ${ }^{1}$ Department of Computer Science, Education College, University of Kufa, Iraq \\ ${ }^{2}$ Department of Computer Science, Faculty of Computer Science and Mathematics, University of Kufa, Iraq
}

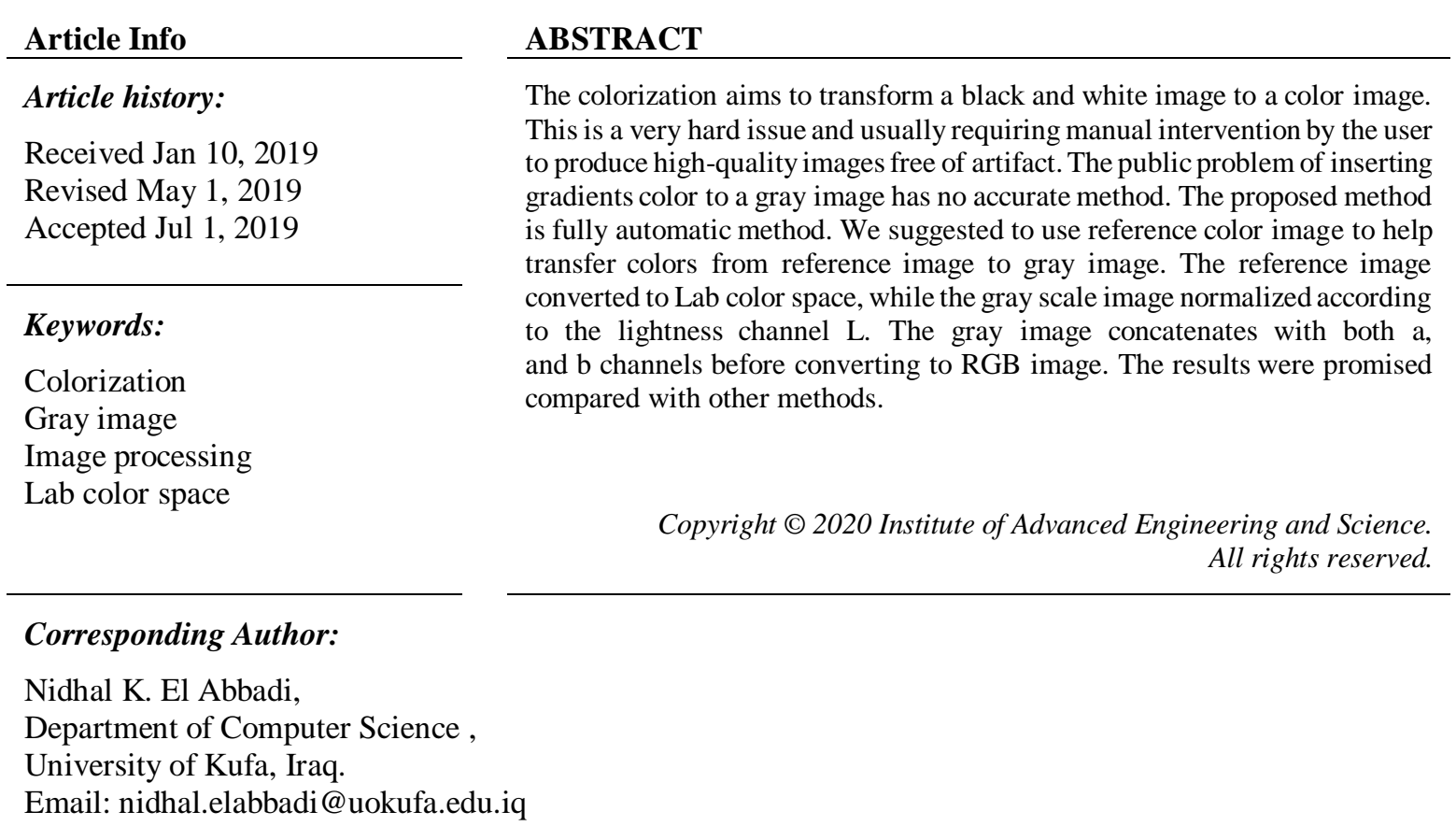

\section{INTRODUCTION}

Image colorization is the operation of inserting colors to black and white images without prior knowledge about the origin colors of objects in the image. The main problem is no accurate solution can achieve from colorizing gray image, this is due to the possibility of various colors values can be assigned to the same luminance with different hue or saturation [1]. We should know that the image we rely on in the color transfer process is rich in colors, so it should be appropriate with gray image scenes. Such problem stimulates researchers to create a novel algorithm, which deem the distribution of colors in the aim image in addition to the target color value during the colorization process [2].

In many cases, colour manipulation may also be necessary to reduce differences between images for further processing. When stitching a panorama, for instance consecutive images may have slight colour variations, hindering the stitching process. Similarly, differences between individual camera sensors may lead to small changes across astereo pair that could affect viewer comfort. Inanother scenario, when processing video content, colour edits applied to one frame often need to be replicated to subsequent frames of a sequence. In each of these scenarios, as well as in creative applications, editing the colour content of images requires skilled and extensive user input, while the tools available to non-expert users tend to not offer adequate control [3]. The main purpose is to make the gray images attractive by the viewer so that the colors are added to be appropriate to scenes the picture. The coloring process works to colorize each pixel in the gray image in a certain color. Coloring methods can be divided into three tyeps: hand coloring, whis is a very extensive human effort is required. Mostly image editing software like paint or adobe Photoshop are used for converting gray image to colored image but this process is very time consuming [4]. The second type is scribble-based colorization and Example-based colorization. The scribble-based methods used in this type need a hard effort by the user to add a great scribble to the gray images. Example-based colorization in this type, the user is 
required to provide a similar reference image suitable for the gray image taken from the similarities of images, and the color convert technologies [5]. Second type the coloring method depends on pixel density and adjacent pixel stats to pixels that are similar to the suggest image and then transform the color of the correspond pixel to the gray pixel, it is later improved in [6] by relying on the texture characteristic. In order to help the user easily get the image that is suitable for coloring, the web-supplied example(s), by using images available on the Internet [7]. Relying on the images available on the Net can be powerful for the lighting teams between the gray image and the suggest image, but requires that the images available contain similar objects and scenes for accurate per-pixel recording between the suggest images and the target image.

Moreover, it is a great idea to paint realistic colors whenever the gray image contains signs and scenes more commonly the colors are more accurately painted. For example, the sky is often blue and the grass is often green and also has many situations set the gray image can map to multiple colored images [8]. Where the problem lies in the creation of a three-layer color image of a gray image consisting of a single layer without prior knowledge of the original colors of the image and without human intervention and that the image is free of artifacts that are considered as noise. There are a number of methods used in this field, some of which we discuss here. Okura et al. [6] Training networks on a group of images use the method of coloring where they are fully trained where different images include the largest number of scenes that can be colored, The images that are trained are taken in closed or outdoor places, whether they are day or night, including pictures that contain local features such as human skin. Irony et.al [7] provides an algorithm for adding colors to a picture based on a standard conversion model of colors from a reference picture to gray picture. Follow this way the techniques of learning under supervision to classify the feature space. The main disadvantage of this scheme is that it required manual segmentation of reference image, which does not make it automatic. T. Welsh [8] introduced method based on the transfer of colors from the suggest image to the grayscale image. The algorithm used to unify color and texture transfer and the algorithm is shown by transferring time changes between images, for example, leaves when trees are empty. Tai et al. [9] suggested a public color converted algorithm. They use Gaussian mixture model for probabilistic segmentation of both reference image and gray image. The authors utilize only lightness value for grayscale image segmentation. The main constraint of their approach is that source image needs to be selected by user. Hu et al. [10] presented a method based on seed pixel selection to support the users in deciding what pixels are highly desired to be colorized for an aloft fineness colorized image. They begin by dividing the grayscale image into non-overlapping windows, and then, for each window, two pixels that convergent the average luminance of window are chosen as the seeds, Then the seed is colored by the user, where they used optimization reduces the variation between the seeds and their neighbouring pixels that used to deploy the colors to the other pixels. $\mathrm{Li}$ et al. [11] proposed method for coloring gray images by using the automatic outcome-finding feature with outcomes combined through Markov Random Field (MRF) model to get better coloring.

Looking them symmetrical or asymmetrical image where vectors are used and then is determine for each local area of the gray image is selected in order to destination the coloring results. Therefore, use a descriptor depend on a luminance deviation to determine whether the area may be symmetrical or asymmetrical. Zhang et al. [12] Instead of selecting colors individually, a colored map is created and then a color palette is used to color the target image. These are the most common methods of coloring since the beginning of the process of coloring gray images, but the progress of the years began to develop gradually, they have become the use of neural networks that produce effective results but need time whenever the database that contains large data are better and more time, and in some ways which use the neural networks have a specific base. In addition to the images contain features similar to the gray image here we touch on some of them: Lizuka [13] colorization the grayscale images by creating a representation of the texture dimension, which represents the color image that is used as a reference image by training a set of SVMs on the input image and thus transferring the colors. Cheng et al. [14] suggested a completely automatic way in which multiple features are taken to colorize various spot of the grayscale image based on a tiny NN. The resulted image improved by using Joint bilateral filter. Al Azzeh et al. [15] Deep learning is used in coloring, where a million images are associated with high-level indications with a target image to guide the user towards effective input to produce good results. Recently, deep learning techniques have achieved impressive results in modeling large-scale data. Image colorization is formulated as a regression problem and deep neural networks are used to directly solve it [16-19]. These methods can colorize a new photo fully automatically without requiring any scribbles or reference. Unfortunately, none of these methods allow multi-modal colorization [20].

The rest of this paper include describing the material and methods used in the suggested method in section 2. While Section 3 display the results when implement the suggested method. And finally in section 4 we conclude and discuss the results and the contribution of this work. 


\section{MATERIAL AND METHODS}

\subsection{Color Space CIEXYZ}

The first types to be used as a chromatic space of specific quantity between the wavelength distributions of the visible spectrum and the colors perceived in the vision of human color. This color space was derived by collecting the experimental results of the color space specifications CIERGB, Figure 1 shows CIE XYZ color space.

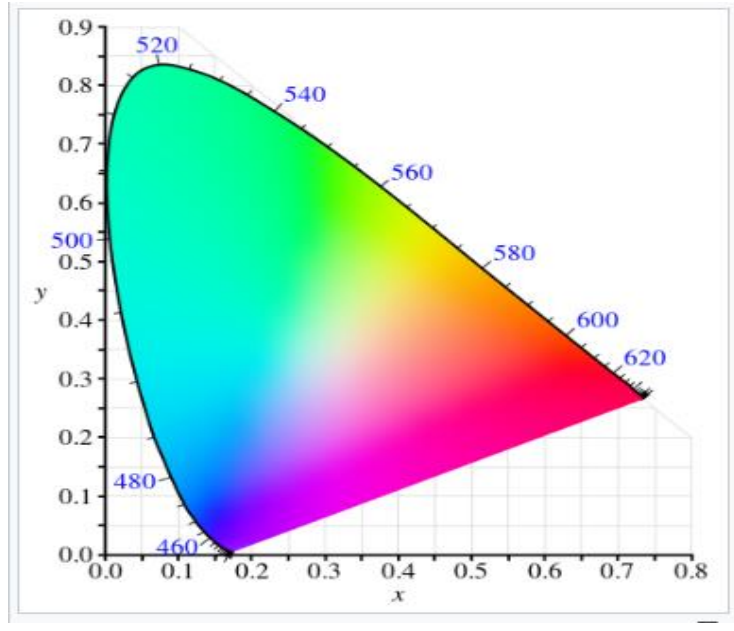

Figure 1. CIE XYZ color model

Converting RGB to CIEXYZ is applied by forward transformation as described in the following [21]:

$$
\left[\begin{array}{l}
X \\
Y \\
Z
\end{array}\right]=\frac{1}{0.176,97}\left[\begin{array}{ccc}
0.4900 & 0.31000 & 0.20000 \\
0.17697 & 0.81240 & 0.01063 \\
0.00000 & 0.01000 & 0.99000
\end{array}\right]\left[\begin{array}{l}
R \\
G \\
B
\end{array}\right]
$$

Where R, G, B are the red, green, and blue pixels of color image. The invers of the CIEXYZ:

$$
\left[\begin{array}{l}
R \\
G \\
B
\end{array}\right]=\left[\begin{array}{lcc}
0.41847 & -0.315866 & -0.082835 \\
-0.091169 & 0.25243 & 0.015708 \\
0.00092090 & -0.0025498 & 0.17860
\end{array}\right]\left[\begin{array}{l}
R \\
G \\
B
\end{array}\right]
$$

This color space consists of all the color sensations that can be seen by the middle-sighted people, and this is why the colors are represented consistently as the basic colors used in real colors, in other words cannot be generated in any light spectrum.

\subsection{CIE L*a*b* (CIELAB)}

A developed model of CIEXYZ color space. It displays all the visible colors of the human and is building to act as an independent model. The three coordinates for Lab were L represent lightness, where (0-represent color black and 100 represent color white), the (a) gradient represents a green and magenta color (range from -128 to 128), where negative values represented green, while positive values represented magenta, the (b) gradient represents between blue and yellow (range from -128 to 128), where the negative values represented the blue color and the positive values was yellow color, Figure 2 shows CIE L*a*b* color space with different lightness [22].

The singular aim of the $\mathrm{L}^{*} \mathrm{a} \mathrm{b}^{*}$ model is to exist 'device-autonomous'. The colors must not be based on the device they are show on. It is a simply notional color model that is utilized at times as an absolute criterion to compare all other color models.

There are no simple formulas to convert RGB color to Lab color model, so we must first transform RGB to a definite color model as CIEXYZ then to Lab color space. Apply the CIELab:

$$
L^{*}=116 g\left(\frac{Y}{Y_{n}}\right)-16
$$




$$
\begin{aligned}
& a^{*}=500\left(g\left(\frac{X 1}{X 1_{n}}\right)-g\left(\frac{Y 1}{Y 1_{n}}\right)\right) \\
& b^{*}=200\left(g\left(\frac{Y 1}{Y 1_{n}}\right)-g\left(\frac{Z 1}{Z 1_{n}}\right)\right) \\
& G(t)=\left\{\begin{array}{cc}
\sqrt[3]{t} & \text { if } t>\sigma^{3} \\
\frac{t}{3 \sigma^{2}}+\frac{4}{29} & \text { otherwise }
\end{array}\right. \\
& X 1=X 1_{n} g^{-1}\left(\frac{L^{*}+16}{116}+\frac{a^{*}}{500}\right) \\
& Y 1=Y 1_{n} g^{-1}\left(\frac{L^{*}+16}{116}\right) \\
& Z 1=Z 1_{n} g^{-1}\left(\frac{L^{*}+16}{116}+\frac{b}{200}\right) \\
& G(t)=\left\{\begin{array}{cc}
t^{3} & \text { if } t>\sigma \\
3 \sigma^{2}\left(t-\frac{4}{29}\right) & \text { otherwise }
\end{array}\right.
\end{aligned}
$$

Where $\sigma=\frac{6}{29}$
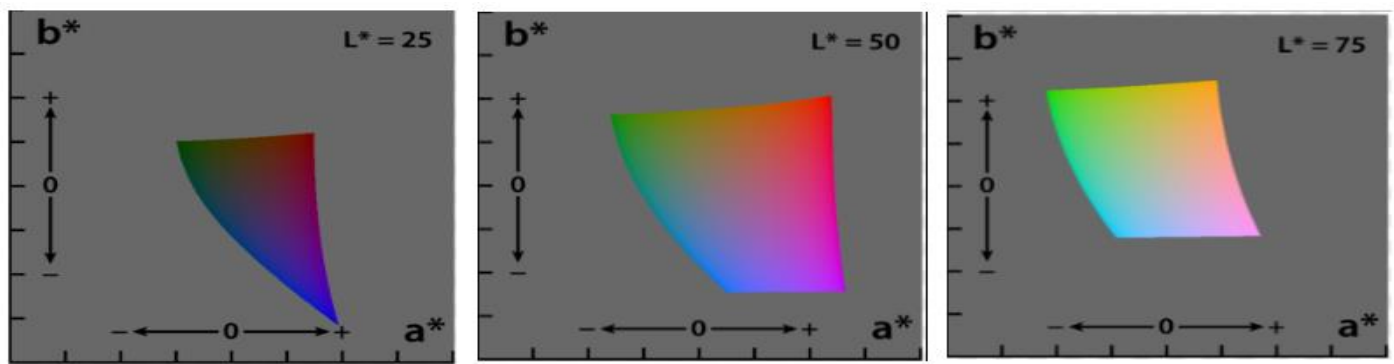

Figure 2. CIE L*a*b* color model when lightness is 25,50 and 75

\subsection{Proposed Method}

Colorization process needs human intervention in determining the color image (reference image) by which the gray image is colored, whether symmetrical or otherwise. The proposed algorithm 1, Table 1 summarized the steps for colorization gray image relies on CIELAB color space.

Table 1. The Proposed Algorithm

\begin{tabular}{ll}
\hline Algorithm 1: Colorization of Image \\
\hline Input: Gray image and RGB image. \\
Output: Colorization of gray image. \\
1. & Select color source image that has similar features to target grayscale image. \\
2. & Convert RGB image from RGB color model into CIEXYZ color model. \\
3. & Convert the resulting image from the previous step to the CIELab color space. \\
4. & Normalize the intensity values for gray image according to L channel of CIELab. \\
5. & Concatenate normalized gray image with (a, b channels) of CIELab. \\
6. & Transform image from CIELab color model to CIEXYZ color model. \\
7. & Convert result of step 6 into RGB color model. \\
8. & Check performance \\
\hline
\end{tabular}

The color image that is selected by the user (reference image) recommend to have similar features to the gray image to be colored. The color image is converted into a CIEXYZ color space that has a specific range of visible colors and then converts the resulting image from CIEXYZ color model to CIELab color model, Figure 3 show the flow chart of proposed algorithm. 
Science truth $94 \%$ of the cells in our eyes mark brightness. That remained $6 \%$ of our receptors to represent as sensors for colors. For that, we focus in this proposal on the similarity of lightness (L) from CIELab color space and gray image. The range of the gray image intensity values normalized to be similar to the range of lightness (L channel) in the CIELab, this can be achieved by using formula 11.

$$
\text { Normalization }=\frac{L_{\max }}{f_{\max }-f_{\min }} *\left(f-f_{\min }\right)
$$

Where $L_{\max }$ is the maximum value of $L$ in Lab color space, $f_{\max }$ is the maximum value in the gray image $(f)$, $f_{\min }$ is the minimum value in gray image $(f)$. The normalized gray image will be concatenate with both ( $\mathrm{a}, \mathrm{b}$ channels of CIELab color space). The result image converts to CIEXYZ color space, and then convert to RGB image, which is the colored image of grayscale image.

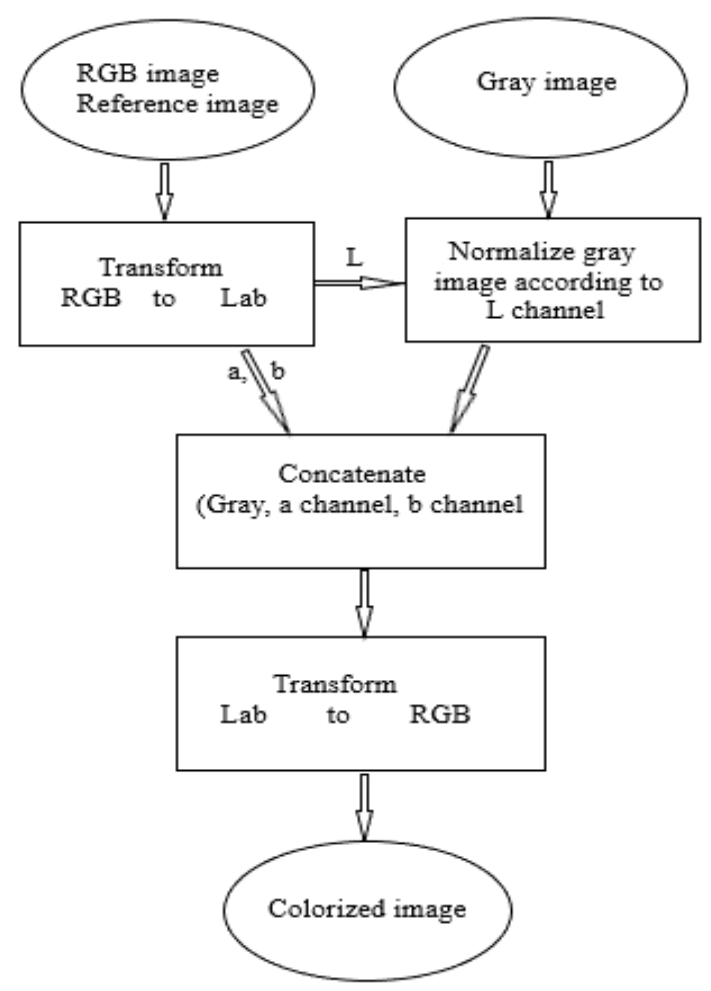

Figure 3. Flow chart for proposed algorithm

\section{RESULTS AND DISCUSSION}

The proposed algorithm implemented with many gray scale image to test the algorithm performance, the performance measured by using PSNR and visual effect. For accurate measuring of colorization performance, we suggested to test gray image converted from known colored image, then compare the colorized image with ground truth image visually, and by determining the PSNR. Selecting the reference image has highly impact on the colorization process. The (RGB) color histogram of reference image has high effect on the colorization process, and the color histogram for the colorized image. The color histogram has direct effect on the range values of both ( $a$, and b) which convey this effect to the colorized image. This showed when colorizing gray image appear below the Figure 4 with different reference images that has different color histogram appear below the Figure 5. It results show the effect of colors in reference images on the colorized images.

\subsection{Measuring PSNR}

1. Mean Square Error (MSE): It is representing important parameter to measure the PSNR, and then the quality of the colorized image. Its represent by:

$$
M S E=\sum_{i=1}^{N} \sum_{j=1}^{M}(C(i, j)-I(i, j))^{2} / M * N
$$


Where $C$ is colorized image, $I$ is gray image, and $(N \times M)$ represent image size.

2. Peak Signal-to-Noise Ratio (PSNR): Is a measure used to measure the proportion of the similarity or extent of the difference between two images of the same structure as shown in Table 1. Its determined by:

$$
P S N R=10 \log 10\left(M A X^{2} / M S E\right)
$$

The PSNR indicate how the colorized image similar to the ground truth image appear below the Figure 6. We conclude that the best suggested image is the image consist of like objects as the target image as shows in Figure 6 image 4.
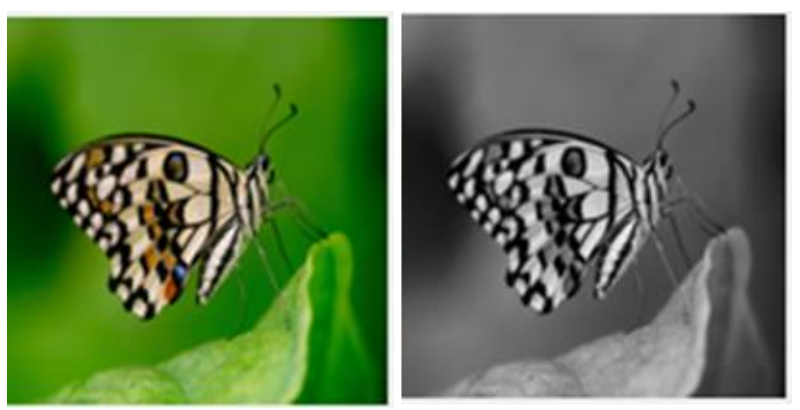

Figure 4. Ground truth image on the left and the gray image on the right

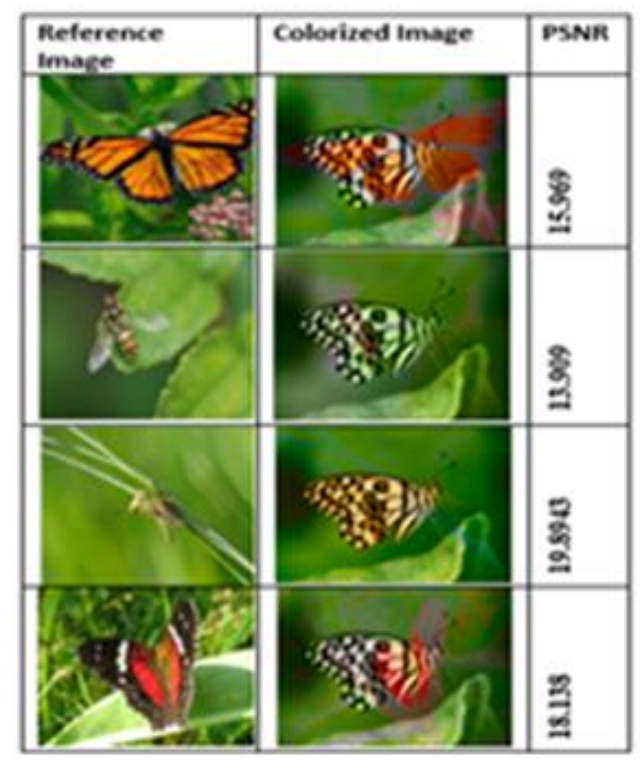

Figure 5. Effect of reference image on colorization process

The last test is colorizing different images with different categories as appear in the Figure 7, which show the robust of suggested method for colorizing any image. Figure 7 showed that reference images with almost similar objects with gray image gives better results than others. Also clear when the color histogram (or colors contains in general) in the reference image has noticed different with colors in gray image, then the result will be with less quality.

The proposed algorithm compared with other algorithm suggested by Cheng et al. the result of applying this algorithm on some of images showed in Figure 8, the same images colorized by proposed algorithm showed in Figure 7. It is clear that the results are almost the same. Also the proposed algorithm is compared with algorithm suggested by Attea et al. and the results showed in Figure 9. 


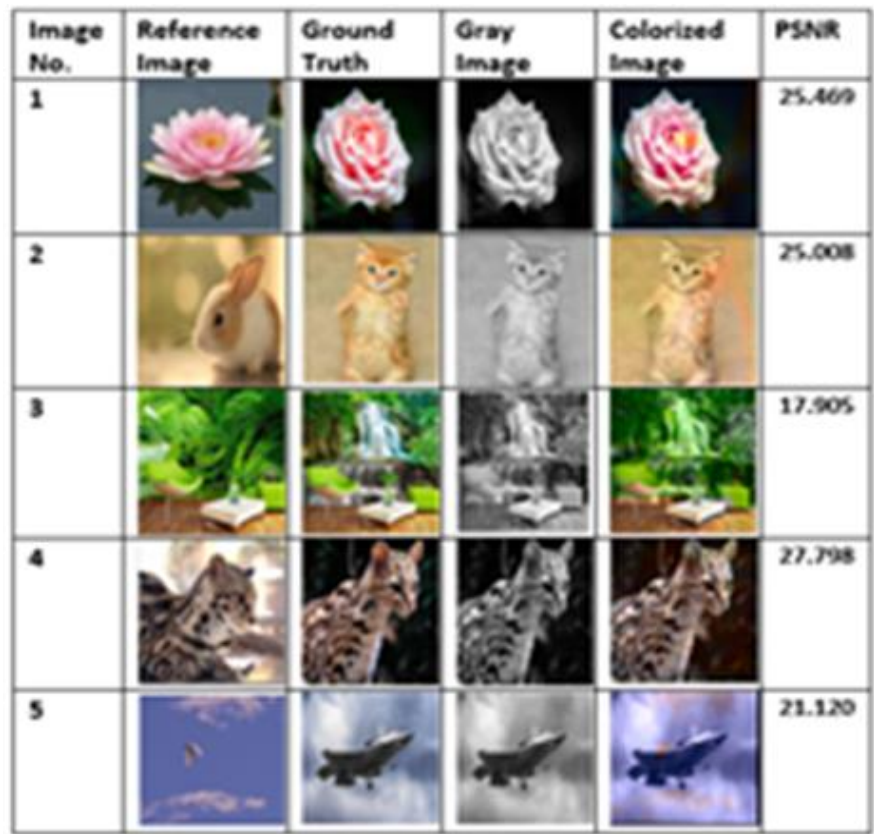

Figure 6. the PSNR for different image colorized by proposed method

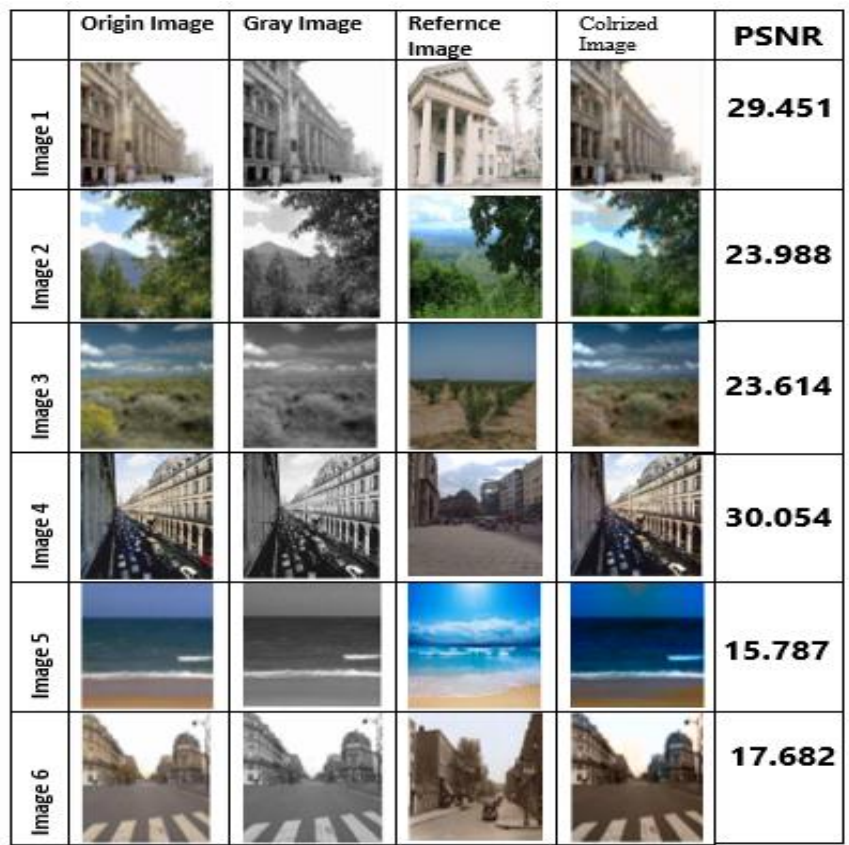

Figure 7. Colorizing images from different categories

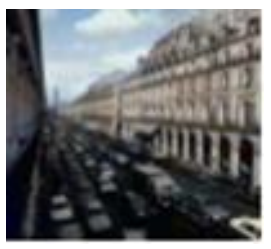

$30 \mathrm{~dB}[14]$

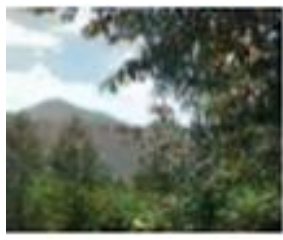

$23 \mathrm{~dB}[14]$

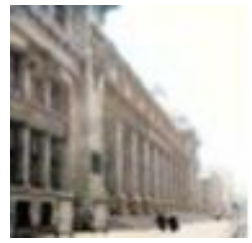

$29 \mathrm{~dB}[14]$

Figure 8. Colorizing images for Cheng et al [14] 


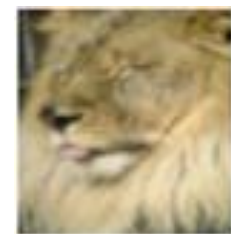

Ref. image

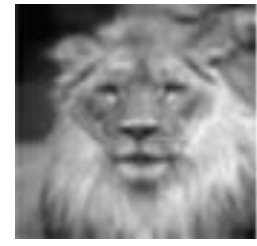

Gray image

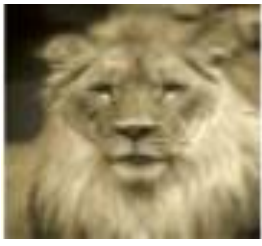

Attea et al. [17]

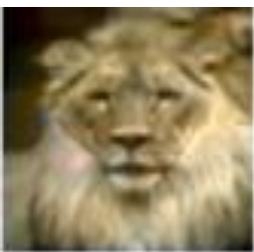

Our method

Figure 9. Comparison results between the proposed colorization and Attea et al [23]
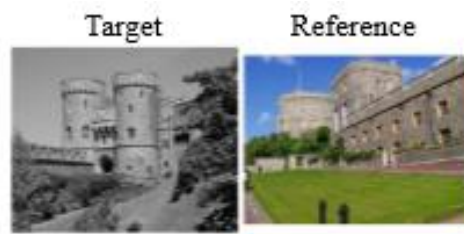

$[24]$
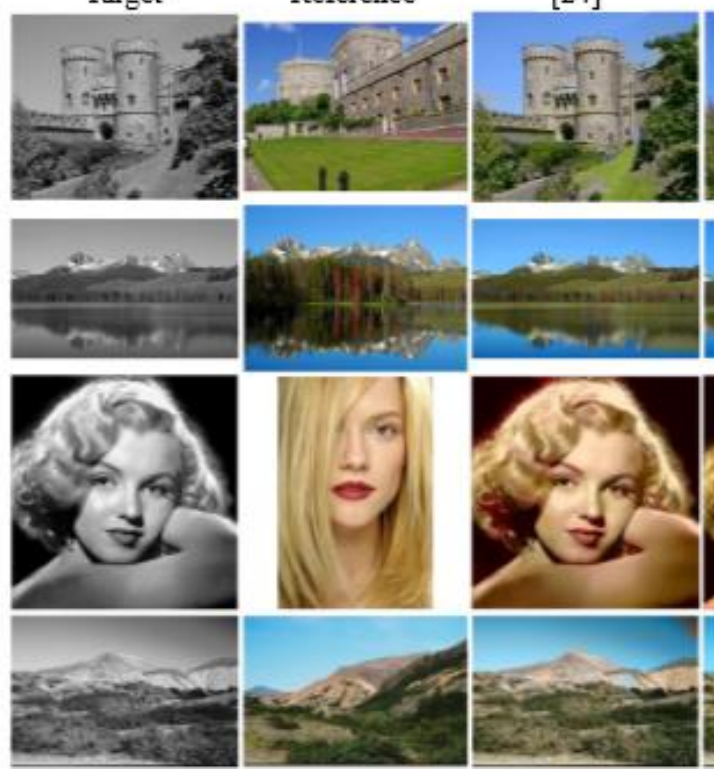

[25]
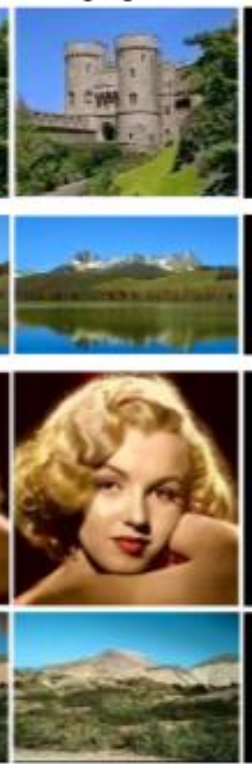

[26]
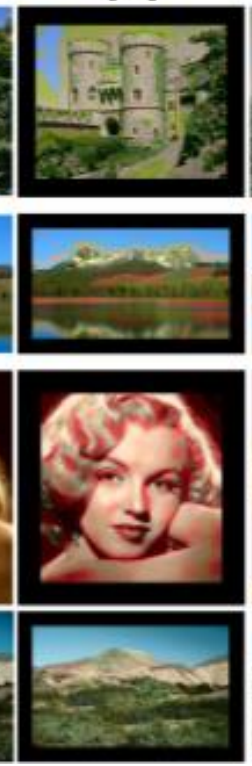

$[27]$
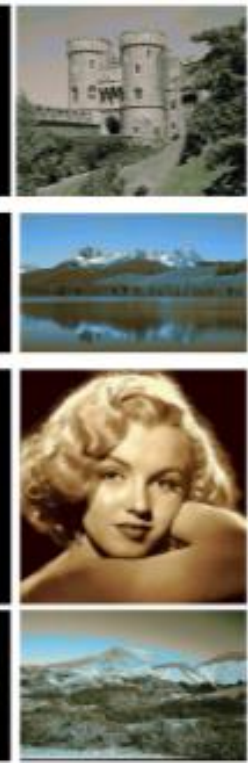

Figure 10. Example results for the colourization methods

\section{CONCLUSIONS}

We presented fully automatic method for colorizing grayscale images depend on Lab color space, and reference image. This method normalized gray image values according to Lightness of Lab model for color image. The proposed method gives promised results (visually and by determine PSNR), also we get good results when compared to the other works. The cornerstone of this method is the need for a reference image containing the same content in the gray image with similar color histogram, it is clear that the colorization of gray image depends on the colors in reference image as in Figure 6, also selecting reference image with same categories as in gray image gives enhance the colorizing process as in Figure 7 . The proposed method is fast to implement and very simple. We suggest for future works to find algorithm which determine suitable reference image from many suggested images.

\section{REFERENCES}

[1] Abdul-Wahab Sami Ibrahim, and Hind Jumaa Sartep, "Grayscale image coloring by using YCbCr and HSV color spaces," International Journal of Modern Trends in Engineering and Research, Volume 04, Issue 4, pages 130-136, 2017. DOI:10.21884/IJMTER.2017. 4130.XD635.

[2] Sou-Young, Jin Ho-Jin Choi, Yu-Wing Tai, "A Randomized Algorithm for Natural Object Colorization," Eurographics, Volume 33 (2014), Number 2, 2014. DOI: 10.1111/cgf.12294.

[3] [3] H. Sheikh Faridul1, T. Pouli, C. Chamaret, J. Stauder, E. Reinhard, D. Kuzovkin, A. Tremeau, "Colour Mapping: A Review of Recent Methods, Extensions and Applications," Computer Graphics Forum, Volume 35, number 1, pp. 59-88, 2016.

[4] [4] Upasana Bisht, Tushar Patnaik, "Overview of Automatic Image Colorization Schemes," International Journal of Advanced Engineering and Global Technology, Vol-03, Issue-10, pp. 1283 -1287, November 2015.

[5] A. Hertzmann, C. E. Jacobs, N. Oliver, B. Curless, and D. H. Salesin. "Image analogies," In Proceedings of the 28th Annual Conference on Computer Graphics and Interactive Techniques, SIGGRAPH ’01, pages 327-340, 2001. 
[6] Okura, Fumio, et al. "Unifying color and texture transfer for predictive appearance manipulation," Computer Graphics Forum. Vol. 34. No. 4. 2015.

[7] R. Irony, D. Cohen-Or, and D. Lischinski. “Colorization by example In Eurographics Symp on Rendering," volume 2. Citeseer, 2005.

[8] T. Welsh, M. Ashikhmin, and K. Mueller," Transferring color to grayscale image," in ACM Transactions on Graphics (TOG), vol. 21, no. 3, pp. 277-280. ACM, 2002.

[9] Tai, Y., Jia, J., Tang, C., "Local Color Transfer via Probabilistic Segmentation by Expectation Maximization," Computer Society Conference on Computer Vision and Pattern Recognition, IEEE, Vol 1 pp. 197-202,2005.

[10] Hu, Min, Bo Ou, and Yi Xiao, "efficient image colorization based on seed pixel selection," Multimedia Tools and Applications, Vol. 76, Issue 22, pp. 23567-23588, 2017, DOI: 10.1007/s11042-016-4112-9.

[11] Li, Bo, Lai, Y. \& Paul L. Rosin. "Example-based image colorization via automatic feature selection and fusion," Neurocomputing 266: 687-698, 2017

[12] Zhang, Richard, et al., "Real-time user-guided image colorization with learned deep priors," arXiv preprint arXiv:1705.02999 (2017).

[13] Iizuka, Satoshi, Edgar Simo-Serra, and Hiroshi Ishikawa., "Let there be color ! : joint end-to-end learning of global and local image priors for automatic image colorization with simultaneous classification," ACM Transactions on Graphics (TOG) 35.4 (2016): 110.

[14] Cheng, Zezhou, Qingxiong Yang, and Bin Sheng., "Deep colorization," Proceedings of the IEEE International Conference on Computer Vision. 2015.

[15] Al Azzeh, Jamil, et al., "Creating a Color Map to be used to Convert a Gray Image to Color Image," International Journal of Computer Applications (0975-8887) 153 (2016).

[16] Deshpande, A., Rock, J., Forsyth, D., “Learning large-scale automatic image colorization,” In Proc. ICCV, 567- 575, 2015.

[17] Larsson, G., Maire, M., Shakhnarovich, G., "Learning representations for automatic colorization”, In Proc. ECCV, 577-593, 2016

[18] Zhang, R., Isola, P., Efros, A. A. 2016. “Colorful image colorization,” In Proc. ECCV, 649-666.

[19] Isola, P., Zhu, J.-Y., Zhou, T., and Efros, A. A., "Image-to-image translation with conditional adversarial networks," In Proc. CVPR, 2017.

[20] Charpiat, G., Hofmann, M., Scholkopf, B., “Automatic image colorization via multimodal predictions,” 126- 139, 2008.

[21] Smith Thomas; Guild, John, "The C.I.E. colorimetric standards and their use," Transactions of the Optical Society, Vol. 33, No. 3, DOI: 10.1088/1475-4878/33/3/301 (1931-32).

[22] Margulis, Dan, "Photoshop Lab Color: The Canyon Conundrum and Other Adventures in the Most Powerful Colorspacem,” Berkeley, Calif.: London: Peachpit; Pearson Education. 2006.

[23] Attea, Bara'A. Ali, Sarab Majeed Hameed, and Aminna Dahim Aboud. "Content-based Grayscale Image Colorization," Proc. CSIT, 2006.

[24] Kuzovkin D., Chamaret C., Pouli T., "Descriptor-based image colorization and regularization. In Computational Color Imaging", 5th International Workshop, CCIW 2015, Saint Etienne, France, Proceedings, pp. 59- 68, 2015.

[25] Gupta R.K., Chia A.Y.-S., Rajan D., Ng E.S., Zhiyong H.: “Image colorization using similar images," In Proceedings of the 20th ACM International Conference on Multimedia, ACM, pp. 369-378, 2012.

[26] Charpiat G., Hofmann M., Sch OOlkopf B., “Automatic image colorization via multimodal predictions,” In Proceedings of the 10th European Conference on Computer Vision: Part III (ECCV '08) (Berlin, Heidelberg), Springer-Verlag, pp. 126-139, 2008.

[27] Welsht., Ashikhminm., Muellerk., "Transferring color to grey scale images," ACM Transactionson Graphics 21, 3, pp. 277-280, 2002. 